\title{
Geomorphologische Untersuchungen im östlichen Kroumir- bergland (Nordtunesien, Gebiet östlich von Tabarka)
}

\author{
Karl-Ulrich Brosche, Hans-Georg Molle \& Georg Schulz*)
}

Geomorphological development, phases of landscape evolution, radiocarbon-datings, sequences of sedimentation, soil erosion, Holocene, Upper Pleistocene,

North Tunisia

$\mathrm{Ku} \mathrm{rz}$ f a s s u $\mathrm{n}$ : Im nordtunesischen Untersuchungsgebiet (östliches Kroumirbergland) lassen sich verschiedene Ablagerungen voneinander trennen. Am jüngsten ist eine Feinmaterialakkumulation, die eine maximale Höhe von 5-6 m über Niedrigwasserbett und ein Erosionsniveau bei $2-2,5 \mathrm{~m}$ aufweist. Diese Akkumulation entspricht den Feinsedimentkörpern ("Auelehme") Fregiens (1971). Nach zwei 14C-Daten (Holzkohle) wurden die Sedimente etwa seit 5000 B. P. abgelagert. Ungefähr in den gleichen Zeitraum fällt nach ${ }^{14} \mathrm{C}$-Daten die Ablagerung einer $6-12 \mathrm{~m}$ mächtigen „Dünensandterrasse" im Oued Malah.

Die nächst ältere Ablagerung besteht aus einem Schotterkörper, der vor allem im Talgrund unter den oben genannten Akkumulationen anzutreffen ist. Nach Vergleichen mit Untersuchungsergebnissen in Zentraltunesien scheint ein würmzeitliches Alter der Schotter wahrscheinlich zu sein. Zeitlich nicht einstufen ließen sich kalkzementierte Dünen mit Terra rossa-Schlotten in Meeresnähe bei 12,5-13,5 m ü. M., eine oder mehrere Schotterterrassen mit Felssockeln bei $25-50 \mathrm{~m}$ über Niedrigwasserbett und kalkzementierte Dünen mit roten Bodensedimenten und einzelnen Sandsteinschottern bei $70-90 \mathrm{~m}$, stellenweise sogar bei $130 \mathrm{~m}$ relativer Höhe über Niedrigwasserbett.

[Geomorphological Investigations in the Eastern Part of the Kroumir Mountains (North Tunisia, Region East of Tabarka)]

A bstract: In the study area in northern Tunisia (eastern Kroumir mountains) various deposits may be distinguished. The youngest is a fine material accumulation, with a maximum height of $5-6 \mathrm{~m}$ above low-water level and an erosion level of $2-2,5 \mathrm{~m}$. This deposit corresponds to the fine sediments ("Auelehme") described by Fregren (1971). 2 14C-datings (charcoal) show that sedimentation began about 5000 B.P. 2 other ${ }^{14} \mathrm{C}$-datings show that a $6-12 \mathrm{~m}$ thick "dune sand terrace" was deposited in the Oued Malah at about the same time.

The deposit next in age consists of gravel, found mainly on the valley-floor beneath the deposits described above. A comparison with results from Central Tunisia indicates a probable Würm age. A chronological classification proved impossible in the case of calcified dunes with terra rossa filled pipes, situated near the sea at 12,5-13,5 m above sea-level, one or more gravel terraces on rock foundations at $25-50 \mathrm{~m}$ above low-water level, and calcified dunes with red soil sediments and isolated sandstone gravel at $70-90 \mathrm{~m}$, in places even at $130 \mathrm{~m}$ relative height above low-water level.

Rés u mé : Dans la région de recherches, en Tunisie septentrionale (la partie orientale des montagnes de Kroumirie), on peut distinguer des accumulations différentes. Le sédiment le plus jeune est représenté par une accumulation de matière fine qui se trouve à une hauteur maximale de $5-6 \mathrm{~m}$ au-dessus du niveau des basses eaux et qui est caractérisée par un niveau d'érosion à 2-2,5 m. Cette accumulation correspond aux sédiments fins ("Auelehme“) de Fregien (1971). Selon deux datations au radiocarbone (des échantillons de charbon de bois), la sédimentation a commencé vers 5.000 ans B. P. Deux autres datations, dont les échantillons étaient prises d'une "terrasse de sable de dune" à 6-12 $\mathrm{m}$, dans l'Oued Malah, prouvent que cette terrasse avait été accumulée pendant la même période.

*) Anschriften der Verfasser: Priv.-Dozent Dr. K.-U. B r o s che, 1000 Berlin 41, Grunewaldstr. 35, Institut f. Phys. Geographie der FU Berlin; Dr. H.-G. M o 11 e und Dr. G. S ch u l z, Geomorphologisches Laboratorium des Inst. f. Phys. Geographie der FU Berlin, 1000 Berlin 33, Altensteinstraße 19. 
L'accumulation antérieure aux sédiments fins consiste en pierrailles qu'on trouve surtout au fond de vallée sous les accumulations mentionnées. Après avoir comparé les résultats avec ceux de la Tunisie Centrale, l'âge wurmien des pierrailles semble être semblable. On ne pouvait fixer l'âge ni de dunes calcifiées non loin de la mer à une hauteur de $12,5-13,5 \mathrm{~m}$, contenant des puits remplis de terra rossa, ni d'une ou de plusieurs terrasses de pierrailles avec des socles en roche massive à 25-50 $\mathrm{m}$ au-dessus du niveau des basses eaux, ni de dunes calcifiées avec des sédiments de sol rouges et des pierrailles de grès isolées qui se trouvent à $70-90 \mathrm{~m}$, parfois même à $130 \mathrm{~m}$ de hauteur relative au-dessus du niveau des basses eaux.

\section{Einleitung, Problemstellung und Literaturüberblick}

Mit den folgenden geomorphologischen Untersuchungen wird das Ziel verfolgt, die Grundzüge der Formen- und Sedimentfolge in einem küstennahen Gebiet Nordtunesiens herauszuarbeiten und die hierbei erzielten Ergebnisse mit den Befunden von Bos (1971) aus der Umgebung von Ain Draham und von FreGIEN (1971) im gleichen Untersuchungsraum zu vergleichen. Darüber hinaus erscheint uns auch ein überregionaler Vergleich mit unseren Arbeitsergebnissen in Zentral- und Südtunesien (Molle \& Brosche 1976; Brosche \& Molle 1975, 1976) sinnvoll zu sein.

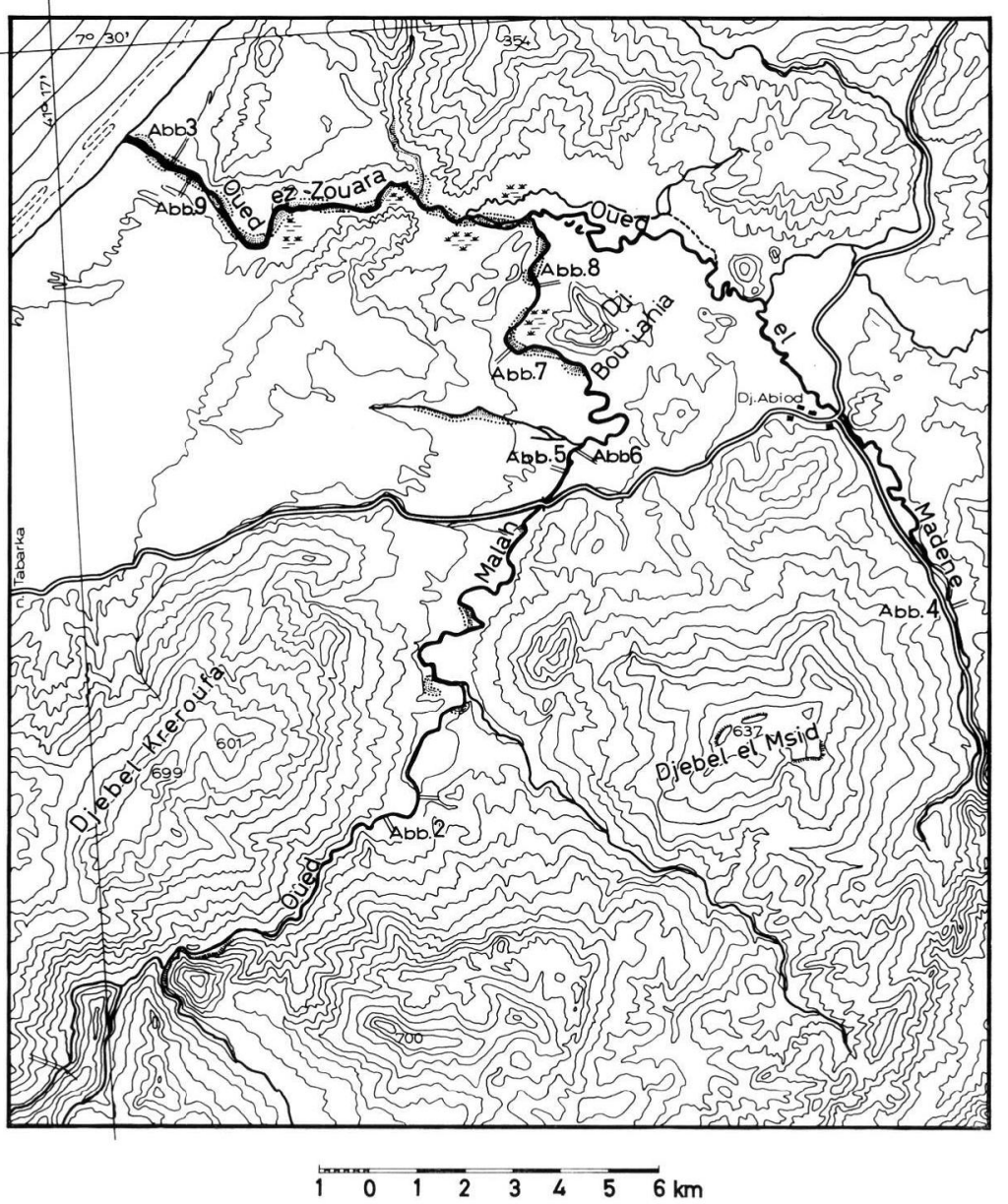

Abb. 1: Übersichtskarte des Arbeitsgebiets. 
Das nordtunesische Arbeitsgebiet liegt östlich Tabarka und umfaßt die Täler des Oued Malah und des Oued el Madene, die sich ca. $8 \mathrm{~km}$ südöstlich der nordtunesischen Küste zum Oued ez Zouara vereinigen (Abb. 1, vgl. auch Karten Nefza, Flle. No. X, 1 : 50000 und Zaouiert Madien, Flle. No. XVII, 1 : 50 000).

Im Raum östlich Tabarka dominieren Kalkmergel, Kalke und Sandsteine des Oligozän, Eozän und der oberen Kreide sowie quartäre, oft durch Kalk zementierte Dünensande. Während im nordöstlichen Matmata-Vorland in Südtunesien und am Djebel Mrhila und am Djebel Chambi in Zentraltunesien markante Schichtstufen- und Schichtkammlandschaften mit weiten Vorlandflächen (z. T. Glacis) die beherrschenden Formenelemente darstellen (Mensching 1958, 1963), bilden die quarzreichen Gesteine im östlichen Kroumirbergland längliche, stark zertalte Schichttafelländer oder schichtkammähnliche, längliche Höhenrücken. Vorlandflächen und Glacis fehlen hier weitgehend.

Auch in klimatischer Hinsicht besteht ein deutlicher Unterschied zwischen dem nordtunesischen Arbeitsgebiet einerseits und den zentral- und südtunesischen Untersuchungsgebieten andererseits. Die Jahresmitteltemperaturen betragen in Tabarka $17,9^{\circ}$, in Zentraltunesien ca. $14,5-19^{\circ}$ (Kairouan 19,1 $1^{\circ}$, Maktar 14,6 $6^{\circ}$ und liegen in Südtunesien bei $20,5^{\circ}$ (Medenine) bzw. 19,2 ${ }^{\circ}$ (Gabes). In Tabarka fallen bei einer etwa dreimonatigen Sommertrockenheit $1047 \mathrm{~mm}$ Niederschlag, in Zentraltunesien dagegen nur $400-800 \mathrm{~mm}$ im Gebirge und 200-400 $\mathrm{m}$ im Gebirgsvorland. Im Matmatabergland und seinem nordöstlichen Vorland fehlen humide Monate. Hier fallen $250 \mathrm{~mm}$ (Matmata) bzw. $178 \mathrm{~mm}$ Niederschlag (Gabes). Den Niederschlagshöhen und der Verteilung der Niederschläge entsprechend bestehen auch hydrogeographische Unterschiede. In Nordtunesien perennieren die Bäche und Flüsse trotz der etwa dreimonatigen sommerlichen Trockenheit im Unterschied zu den erwähnten Gebieten in Zentral- und Südtunesien.

Vor einer Darstellung der Geländebefunde soll kurz auf die Arbeitsergebnisse von FREGIEN (1971) im gleichen Untersuchungsgebiet eingegangen werden. FrEGIEN (1971: 145-148) fand als jüngste Akkumulation in den Tälern des Kroumir- und Mogodberglandes einen Feinsedimentkörper, der dem jüngsten Schotterkörper („unterste Flußterrasse“) aufliegt und von ihm als „Auelehm“ gedeutet wird, der „während der vergangenen rund 3000 Jahre“ in den Ebenen und Tälern gebildet worden sein muß. Bezüglich der zeitlichen Einstufung dieses Sediments stützt sich FrEGIEN nicht auf eigene Datierungen, sondern erwähnt, daß JAUZEIN (1959: 31) in ähnlichen Alluvialkörpern zentraltunesischer Oueds in drei bis vier Meter Tiefe mehrfach römische Töpferwaren gefunden hat. Daneben unterscheidet Fregien (1971: 147) in den Unterläufen aller Oueds zwei, in einigen Oueds (Oued Sedjenane und Oued Malah) drei weitere Terrassen aus groben, gut gerundeten Schottern. Mit dem Begriff „Terrasse“ meint Fregien bei der 1. (untersten) Terrasse nur den Schotterkörper, bei der 2. und 3. Terrasse morphologische Form und Sediment. Der bereits erwähnte Feinsedimentkörper liegt auf der untersten Terrasse, d. h. auf dem untersten Schotterkörper, der seinerseits von den rezenten Oueds mindestens 1,5 m zerschnitten ist. Flächenmäßig weiter verbreitet als die unterste Terrasse ist die zweite Terrasse. Am Oued Malah und am Oued el Madene beträgt der Höhenabstand zwischen der untersten und der zweiten Terrasse kurz vor Eintritt in die Nefza-Ebene $3 \mathrm{~m}$. Die beiden Terrassen zeigen in allen Tälern flußabwärts eine Tendenz zur Konvergenz, ohne aber zu konvergieren, flußaufwärts divergieren sie. Die zweite Terrasse dient wegen ihrer flächenmäßigen Ausprägung als bevorzugte Siedlungs- und Kulturlandfläche. Das Terrassenmaterial der zweiten Terrasse unterscheidet sich nach Geröllgröße und Rundungsgrad kaum von dem der tieferen Terrasse. Es ist aber stärker verbacken (FREgIEN 1971: 148). „Noch höhere Terrassenreste reichen wieder mehrere Meter über das zweite Terrassenniveau hinauf" (FrEGIEN: 148). 


\section{Die Geländebefunde am Oued ez Zouara, am Oued Malah, am Oued el Madene und in benachbarten Gebieten}

a. Die Feinmaterialakkumulation

Neben dem Niedrig- und Hochwasserbett ${ }^{1}$ ) läßt sich im Gebiet östlich Tabarka eine jüngere $\mathrm{Fe}$ in materia lakk umulation erkennen. Bei diesem graubraunen bzw. braunen Feinsediment handelt es sich meistens um sandige und lehmige Ablagerungen, die z. T. im unteren Abschnitt von Kieslagen durchsetzt sind. Die Feinmaterialakkumulation ist eine eigenständige Akkumulation und ist mit Sicherheit mit dem Auelehm bzw. Auesand, den Fregien (1971: 144-146) aus dem Kroumir- und Mogodbergland beschreibt, identisch. Die Feinmaterialakkumulation liegt meistens mit einem abrupten Wechsel locker gelagerten, älteren Sandsteinschottern auf (FREGIEN 1971: 145), bzw. sie legt sich seitlich an diese an (Abb. 2). Der Übergang zu den älteren Schottern an der Basis erfolgt manch$\mathrm{mal}$ in einer mehrfachen Wechsellagerung von Schottern und Feinmaterial oder einem zunehmenden Vorherrschen von Schottern gegenüber dem Feinmaterial. Stellenweise liegt die Feinmaterialakkumulation der rinnenförmig modellierten Oberfläche der älteren Schotter auf. Die Feinmaterialakkumulation läßt sich am Oued Malah vom Beginn des Mittellaufs ${ }^{2}$ ) an ca. $6 \mathrm{~km}$ südlich der Straße Tabarka-Djebel Abiod durchgehend bis ans Meer morphologisch verfolgen (Abb. 3). Im Oued el Madene ist sie gleichfalls vom Oberlauf bis zum Unterlauf verfolgbar. In den Talabschnitten südlich des Ortes Djebel Abiod (Nefza) ist die Feinmaterialakkumulation allerdings nur in Gestalt einer 0,8-1,0 m mächtigen Auelehmdecke entwickelt, die ältere, locker gelagerte Schotter überdeckt (Abb. 4).

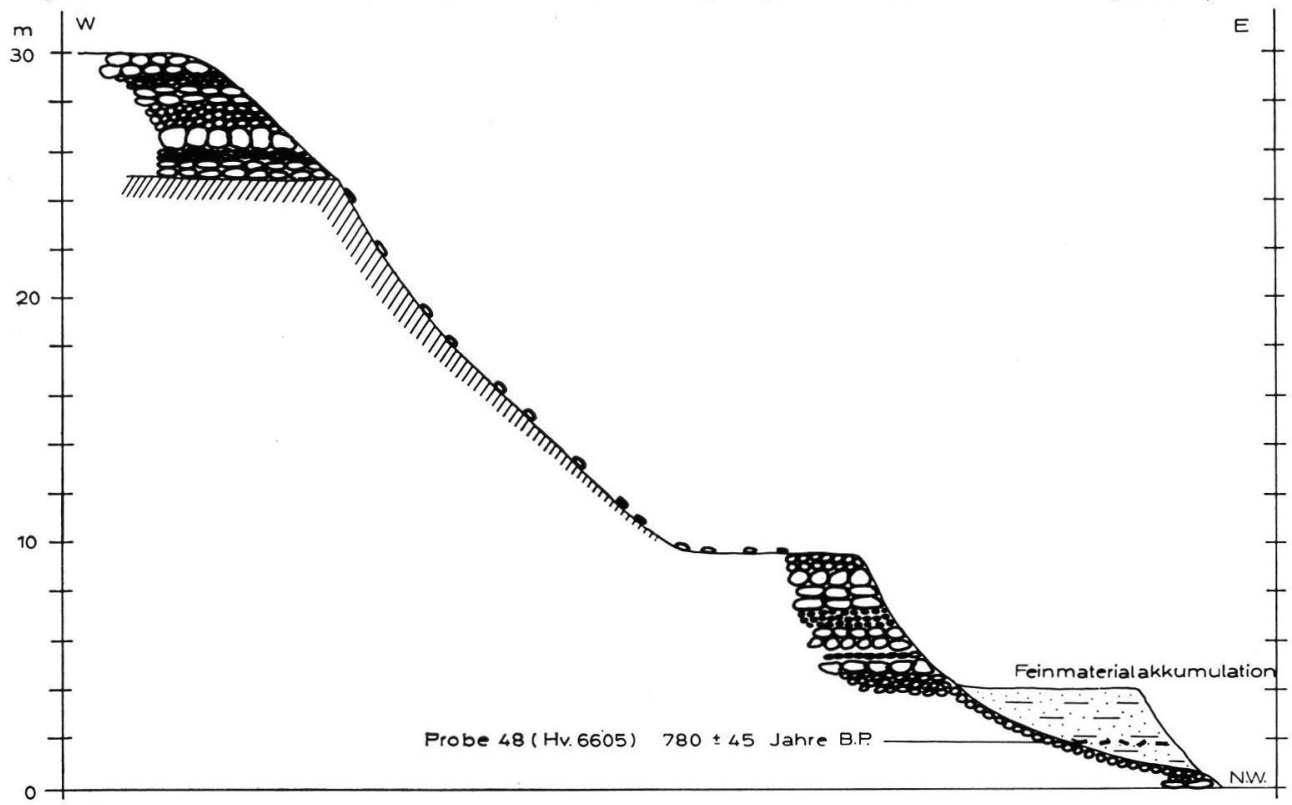

Abb. 2: Anlagerung der Feinmaterialakkumulation an die jüngere Schotterakkumulation am Beginn des Mittellaufes des Oued Malah. Auf einem ca. $25 \mathrm{~m}$ über Niedrigwasserbett gelegenen Felssockel befindet sich eine ältere Schotterakkumulation.

1) Niedrigwasserbett und Hochwasserbett werden in den Abbildungen mit NW und HW abgekürzt.

2) Als Unterlauf wird der Talabschnitt des Oued ez Zouara, als Mittellauf der Talabschnitt des Oued el Madene und des Oued Malah von ihrer Vereinigung zum Oued ez Zouara bis zur Engtalstrecke im Gebirge bezeichnet. Als Oberlauf werden die Engtalabschnitte im Gebirge aufgefaßt. 


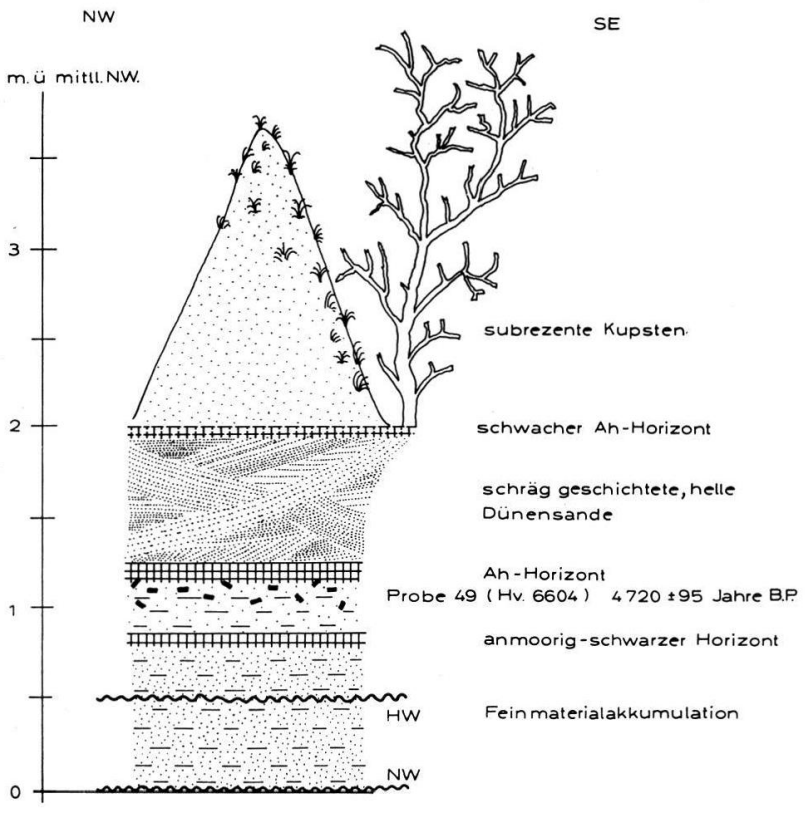

Abb. 3: Sediment- und Bodenabfolge im Bereich der Feinmaterialakkumulation im Unterlauf des Oued ez Zouara.

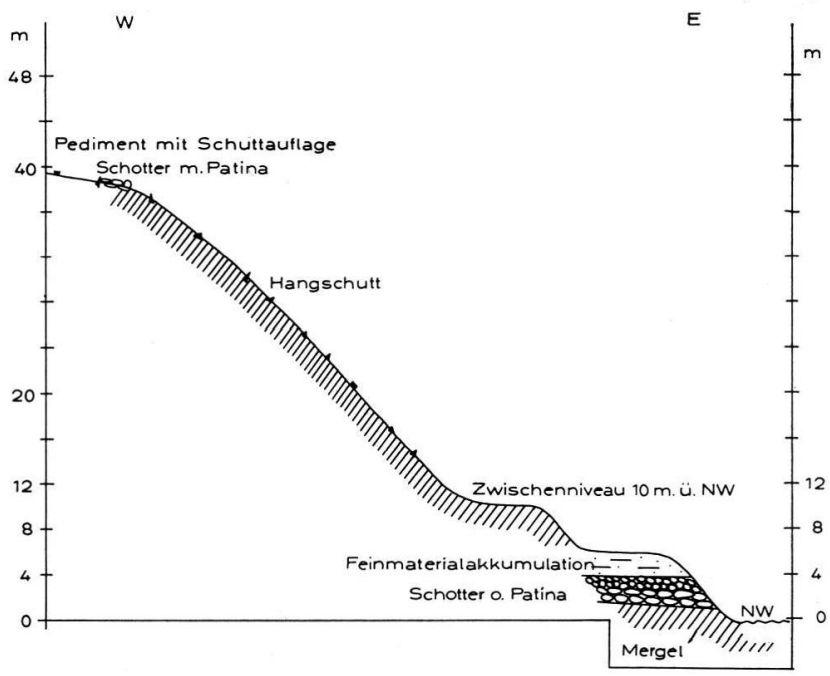

Abb. 4: Die Feinmaterialakkumulation liegt im Mittellauf des Oued el Madene in Gestalt einer Auelehmdecke auf der jüngeren Schotterakkumulation $4 \mathrm{~m}$ über dem Niedrigwasserbett. Links in der Abb. der kurze Rest eines ausgedehnten Pediments mit Resten einer älteren Schotterdecke. 
Die Feinmaterialakkumulation erreicht ihre größte Verbreitung und Mächtigkeit von 4-5 Metern im Talabschnitt nördlich der Straße Tabarka-Djebel Abiod (Abb. 5 u. 6). Bereits am Beginn des Mittellaufes ca. 6 km südlich der genannten Straße kommen Mächtigkeiten von $3,0-3,5 \mathrm{~m}$ vor. In Meeresnähe, wo die Feinmaterialakkumulation ca. $0,8 \mathrm{~km}$ südöstlich der Küste endet, ist sie wenigstens 1,3-1,5 m mächtig. Wie tief sie hier noch unter das Flußbett des Oued ez Zouara hinabgeht, konnte nicht festgestellt werden.

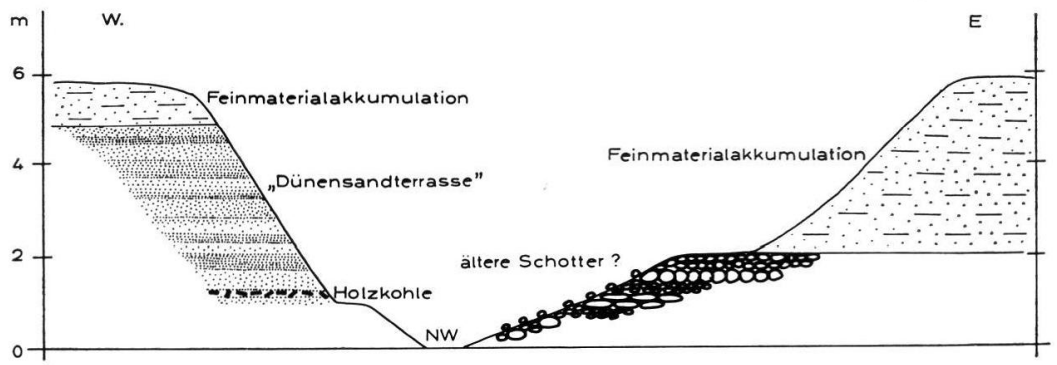

Abb. 5: Feinmaterialakkumulation auf der jüngeren Schotterakkumulation bzw. auf der mit Holzkohlebändern durchsetzten „Dünensandterrasse“ im mittleren Mittellauf des Oued Malah.

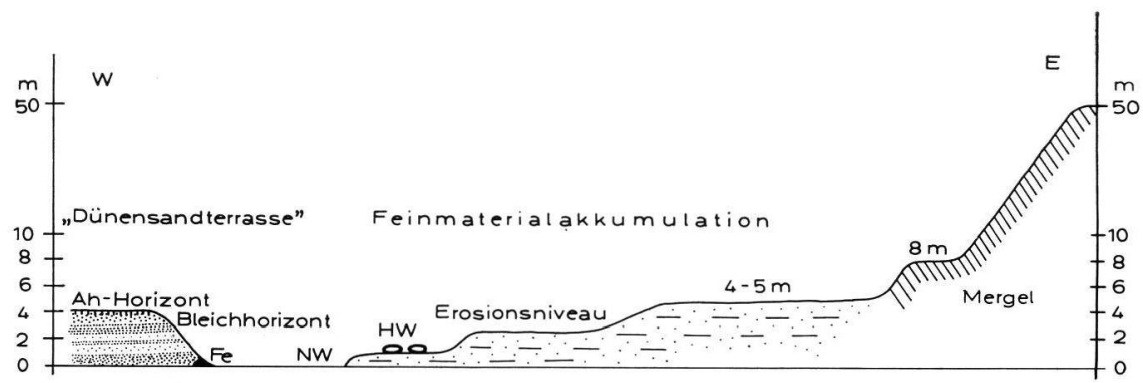

Abb. 6: Feinmaterialakkumulation mit heute nicht mehr überschottertem Erosionsniveau oberhalb des Hoch- und Niedrigwasserbettes des Oued Malah. "Dünensandterrasse" etwa im gleichen Niveau wie Feinmaterialterrasse.

Charakteristisch für die Feinmaterialakkumulation, die - wie in Zentraltunesien die eigentliche landwirtschaftliche Nutzfläche in diesem Gebiet darstellt, ist ihre $\mathrm{Z}_{\mathrm{w}}$ e i g li e d rigkeit (Abb. 6). Oberhalb des Hochwasserbettes ist die Feinmaterialakkumulation oft in zwei Niveaus ausgebildet. Das untere ist als 2-2,5 m über Niedrigwasserbett gelegenes Erosionsniveau entwickelt, das obere liegt $4-6 \mathrm{~m}$ über Niedrigwasserbett und stellt wahrscheinlich die Oberfläche der Akkumulation dar.

Nach Fregien (1971: 146) sollen die Feinsedimentkörper z. T. noch jetzt bei Hochwasser überschwemmt und überdeckt werden. Daraus schließt er, daß es sich bei ihnen „im ganzen um einen rezenten Akkumulationskörper handelt". An einer anderen Stelle (s. o.) äußert Fregien (1971: 145-146), daß die Feinsedimentkörper in den vergangenen rund 3000 Jahren aufgeschüttet worden sein müssen. - Ob Fregien allerdings mit einer rezenten Überschwemmung der von uns festgestellten 5-6 m über Niedrigwasserbett gelegenen Feinsedimente (Abb. 5 u. 6) rechnet, erscheint uns zweifelhaft zu sein, denn nach FrEGIEN (1971: 147) ist das 5-6 m über Niedrigwasserbett gelegene Niveau, das nach seinen und unseren Beobachtungen die Haupt-Siedlungs- und Landwirtschaftsfläche darstellt, die zweite Schotterterrasse. Das allerdings widerspricht völlig unseren Beobachtungen (s. S. 152). 
Im Unterlauf des Oued ez Zouara liegt das untere Niveau in der Feinmaterialakkumulation bei $1,0-1,5 \mathrm{~m}$, das obere bei $3 \mathrm{~m}$ über dem Niedrigwasserbett. Diese Beobachtung entspricht völlig den Beschreibungen FREGIENs (1971: 147), nach denen flußabwärts eine Tendenz des Konvergierens der verschiedenen Terrassen festzustellen ist. Freilich spricht FREGIEN (1971: 147) das obere Niveau in der Feinmaterialakkumulation unserer Meinung nach fälschlicherweise als zweite Schotterterrasse an.

Auf dem 4-6m hohen Niveau der Feinmaterialakkumulation beginnen kleine, $1-1,5 \mathrm{~m}$ tiefe Tälchen mit muldenförmigem Querprofil und zerschneiden es leicht. Sie laufen auf der unteren 2-2,5 $\mathrm{m}$ ü. NW gelegenen Erosionsterrasse in der Feinmaterialterrasse aus. Mit rückschreitender Erosion greifen stellenweise Kerben in diese Muldentäler ein, die vom rezenten Bach ausgehen. Es gibt auch Tälchen, die auf den höheren Hängen beginnen und auf der 4-6-m-Terrasse auslaufen.

Die Feinmaterialakkumulation läßt sich als eine Ablagerung deuten, deren Hauptteil aus Abtragungsprodukten holozäner Bodenbildung entstanden sein könnte. Dafür sprechen die dominierenden Korngrößen (Schluffe, Tone, Sande, vgl. dazu die Korngrößenanalysen von FrEGIEN 1971: 145) und die graubraune bzw. braune Farbe der Sedimente.

Dieser Schluß läßt sich auch durch die Untersuchungsergebnisse von Bos (1971: 83-84 u. 124-125) stützen, der aufgrund seiner Studien in der Umgebung von Ain Draham zu einer Zweiteilung des Holozäns kommt: In dem älteren Abschnitt des Holozäns hat sich hier ein Boden mit braunem $\mathrm{B}_{\mathrm{t}}$-Horizont entwickelt, der im jüngeren Abschnitt des Holozäns einer intensiven Abtragung unterlag. In diesem Zeitraum wurde er degradiert und als Kolluvium an den Hangfüßen und auf flachen Fußflächen abgelagert. Im älteren Abschnitt des Holozäns herrschte nach Bos (1971) eine dichtere Vegetation mit Erlen vor, die heute in diesem Gebiet nicht mehr vorkommt, wie sich aus pollenanalytischen Untersuchungen schließen läßt. Der ältere Abschnitt des Holozäns wird von Bos (1971) u. a. auch aufgrund des Dominierens lehmig-toniger Sedimente als eine wärmere Zeit angesehen, in der die chemische Verwitterung stärker wirksam war als im jüngeren Abschnitt des Holozäns. 14C-Daten der Verfasser (s. u.) werden zeigen, daß sich die von Bos (1971) abgeleiteten Ergebnisse absichern lassen.

Eine ${ }^{14} \mathrm{C}$-Datierung von Holzkohle aus einem ca. $5 \mathrm{~cm}$ mächtigen Holzkohleband, das etwa 0,5 m über der Basis der Feinmaterialakkumulation am oberen Ende des Mittellaufes des Oued Malah gefunden wurde (Abb. 2), ergab ein radiometrisches Alter von $780 \pm 45$ B.P. (Hv 6605). Mit diesem Datum dürfte etwa der Beginn der Feinmaterialakkumulation an dieser Stelle erfaßt sein, der allerdings noch etwas weiter zurückliegen muß, da das Probenmaterial 0,5 $\mathrm{m}$ über der Basis der Feinmaterialakkumulation gesammelt wurde.

Ein weiteres ${ }^{14} \mathrm{C}$-Datum, das am meernahen unteren Ende der Feinmaterialakkumulation gewonnen wurde (Abb. 3), ergab ein radiometrisches Alter von $4720 \pm 95$ B.P. ( $\mathrm{Hv}$ 6604). Bei dieser Holzkohlenprobe, die im $A_{h}$ - und in einem schwach entwickelten $\mathrm{A}_{\mathrm{e}}$-Horizont eines subrezenten Bodens gefunden wurde, handelt es sich um einzelne, recht große Holzkohlestückchen, die vom Fluß verschwemmt wurden. Bei beiden ${ }^{14} \mathrm{C}$-Daten ist allerdings die Möglichkeit nicht ausgeschlossen, daß die Holzkohlestückchen älter sind als die Akkumulation, in der sie gefunden wurden.

Ob die 0,8-1 m mächtigen Feinmaterialdecken, die 4-5 $\mathrm{m}$ über den Niedrigwasserbetten über älteren Schottern liegen (Abb. 4), zeitlich mit den maximal 4-5 m mächtigen Feinmaterialdecken in den weiter talab gelegenen Talstrecken gleichzusetzen sind, läßt sich aufgrund der beiden ${ }^{14} \mathrm{C}$-Daten nicht belegen.

Die zeitlich recht weit auseinander liegenden ${ }^{14} \mathrm{C}$-Alter der beiden datierten $\mathrm{Holz}$ kohleproben in Küstennähe und im oberen Mittellauf des Oued Malah können dafür sprechen, daß entweder die Ablagerung der Feinmaterialakkumulation über einen langen Zeitraum - vielleicht in einem Vorgang rückschreitender Akkumulation - erfolgte oder daß im Zeitraum zwischen ca. 5000 B.P. und nach 800 B.P. eine mehrfache Ablagerung von Feinmaterialsedimenten stattfand. 
Nach ihrer Ablagerung wurde die Feinmaterialakkumulation nicht nur linienhaft durch die Tiefenerosion der Flüsse zerschnitten, sondern es kam in Küstennähe zu einer Überformung (Abb.3), die hier möglicherweise zu einer flächenhaften Erniedrigung der Feinmaterialakkumulation führte. Danach muß eine kräftige Bodenbildung stattgefunden haben, die von einer Phase der Ablagerung von Dünensand abgelöst wurde (vgl. auch die Ausführungen über die „Dünensandterrasse“ im folgenden Kapitel). Diese Phase fand ihren Abschluß mit der Bildung eines schwachen $A_{h}$-Horizonts und einer Besiedlung des Flugsandes durch Baumvegetation. Eine rezente Flugsandverwehung und Dünenbildung folgte, die das Absterben der Bäume verursachte.

Eine ähnliche, allerdings kräftigere Bodenbildung in Dünensanden läßt sich auch östlich Tabarka in der Meknas-Ebene in Küstennähe beobachten. Wir vermuten, daß es sich dabei um die von Fregien (1971: 150) erwähnte fossile Waldbodenbildung handelt, die er als bodenkundliches Zeugnis eines jüngeren "Subpluvials" deutet. Diese nach Fregien auch in der Nefza-Ebene auftretende Bodenbildung soll eine ältere, trockenere Dünenperiode von der jüngeren, noch andauernden Dünenbildung trennen.

\section{b. Die „Dünensandterrasse“}

Nur über eine Strecke von ca. $4 \mathrm{~km}$ ist auf der W-Seite des Oued Malah eine meistens aus feinem Flugsand aufgebaute, 6-12 m hohe Terrasse entwickelt, die wir „D ü n e n s a nd ter rasse" nennen (Abb. 5, 7 u. 8). Diese Terrasse hat ihr Verbreitungsgebiet im Talabschnitt nördlich der Straße Tabarka-Djebel Abiod. Hier konnten zwischen den beiden Höhenzügen Djebel Kreroufa (699 m ü.M.) und Rhoumd er Roumel (215 m ü.M.) von Westen, von der Küste her, mächtige Sandmassen nach E landeinwärts geweht werden (es ist zu vermuten, daß das Oued Malah früher einmal zwischen den beiden genannten Höhenzügen seinen Lauf nach WNW nahm).

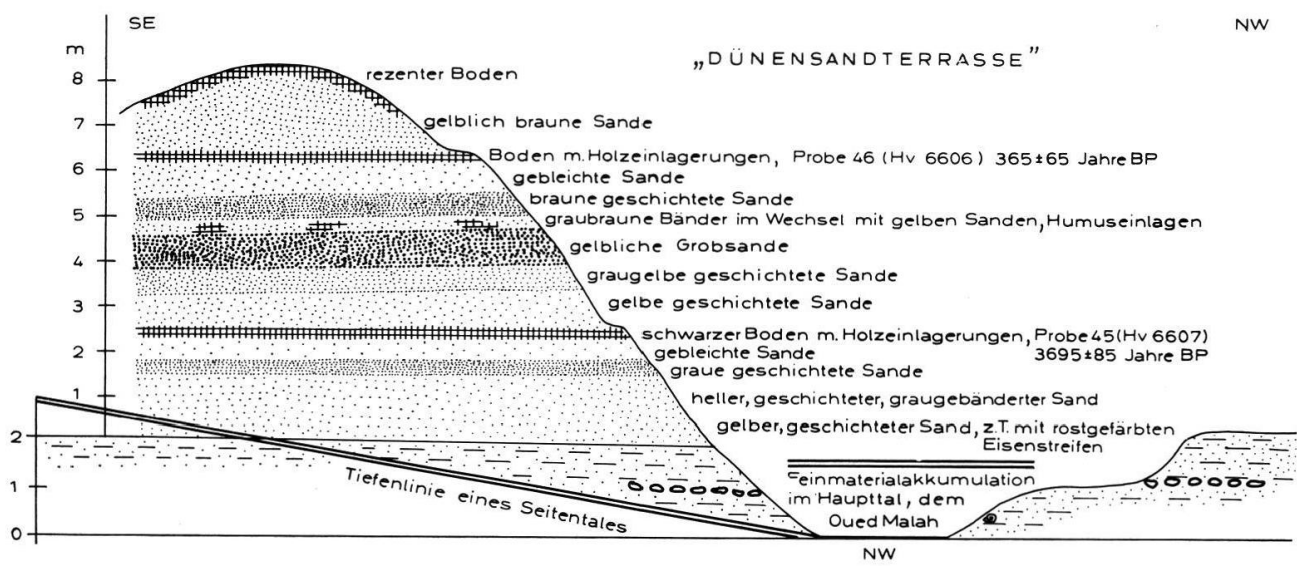

Abb. 7: Polyzyklisch aufgebaute Sedimente der "Dünensandterrasse“ in einem kleinen Nebental des Oued Malah auf Feinmaterialsedimenten. Zwei fossile $\mathrm{A}_{\mathrm{h}}$-Horizonte mit ${ }^{14} \mathrm{C}$-datierten Holzresten in den Dünensanden.

Die „Dünensandterrasse“ besteht, wie oben bereits erwähnt wurde, ganz überwiegend aus feinen, schräg- und kreuzgeschichteten Flugsanden. Nur stellenweise sind einige Kiesund Schotterbänder eingelagert (Abb. 8), die wahrscheinlich fluvial ${ }^{3}$ ) transportiert wur-

3) Eine ähnliche fluviale Verspülung von Schottern und Kiesen in flachen Tälern auf verkalkten Dünensanden läßt sich heute in Nordtunesien in Küstennähe häufig feststellen (z. B. am Cap Serrat). 


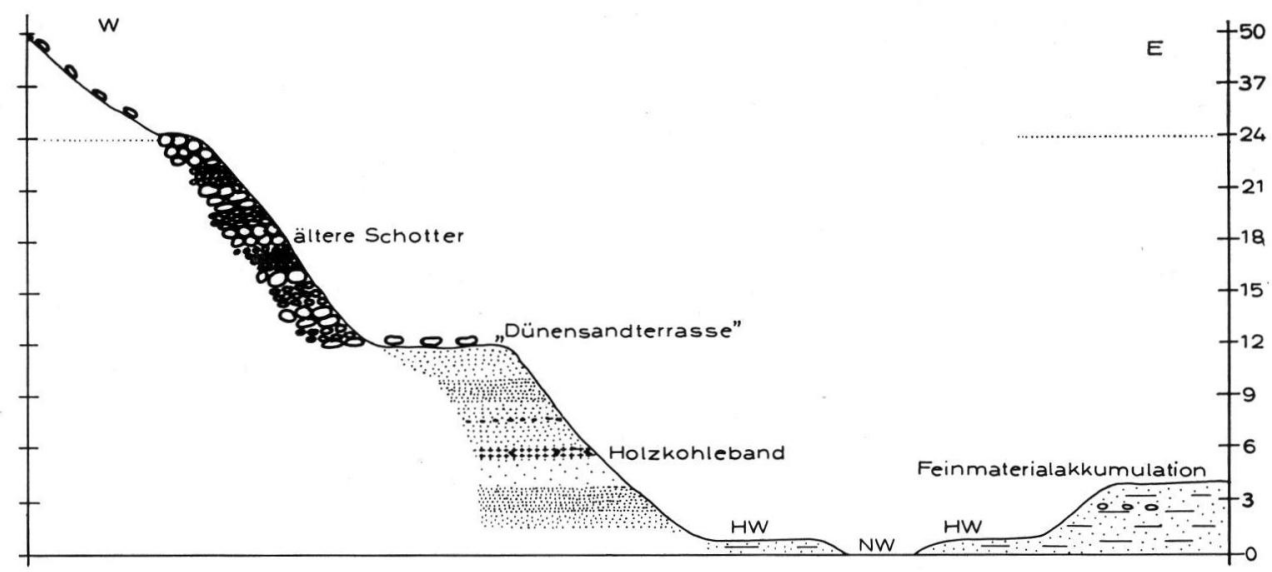

Abb. 8: Feinmaterialakkumulation sowie „Dünensandterrasse“ mit Holzkohleband, die an ältere Schotterakkumulation angelagert ist.

den. Sie stammen - wie einige Schotter und Kiese an der Oberfläche der Dünensandterrasse - wahrscheinlich aus dem älteren, höher hinaufreichenden Schotterkörper.

An einer Lokalität war zu erkennen, daß sich die „Dünensandterrasse“ auf eine ältere Akkumulation legt. Bei dieser älteren Akkumulation handelt es sich um graue Feinsedimente, in die einzelne Kies- und Schotterbänder eingelagert sind.. Es liegen hier wahrscheinlich die unteren Teile der Feinmaterialakkumulation vor. Diese morphologische Einstufung stimmt auch mit den ${ }^{14} \mathrm{C}$-Daten überein, die an Holzproben der „Dünensandterrasse" (Abb. 7) und an Holzkohle aus der Feinmaterialakkumulation in Küstennähe (Abb. 3) gewonnen wurden.

Die „Dünensandterrasse“ liegt tiefer als eine Schotterakkumulation (Abb. 8), an die sie seitlich angelagert ist, und ist daher jünger als diese Schotterakkumulation.

Charakteristisch für die „Dünensandterrasse“ ist die Einschaltung eines (oder mehrere) Holzkohlebänder; in einem Falle streicht ein solches Band in ca. $4 \mathrm{~m}$ (Abb. 8), in zwei anderen Fällen dagegen in nur $2 \mathrm{~m}$ bzw. ca. $1 \mathrm{~m}$ Höhe über dem Niedrigwasserbett aus (Abb. 5). Die Frage, ob es sich um das gleiche Holzkohleband handelt, könnte nur über ${ }^{14} \mathrm{C}$-Datierungen beantwortet werden, da die Fundpunkte zu weit auseinander liegen und die Holzkohlebänder sich im Gelände nicht verbinden lassen. Auf die Datierung der Holzkohlebänder wurde einstweilen verzichtet, da wir die „Dünensandterrasse“ an einer anderen Stelle besser datieren konnten.

In einem kleinen, von W kommenden Nebental des Oued Malah auf der Höhe der Ortschaft Al. el Hammam ist besser als bei den bereits angesprochenen Lokalitäten zu beobachten, daß die "Dünensandterrasse" in mehreren Phasen aufgeweht wurde (Abb.7). Sie ist nämlich durch mehrere Bodenhorizonte $\left(\mathrm{A}_{\mathrm{h}}\right.$ - und $\mathrm{B}_{\mathrm{v}}$-Horizonte), Torf- und Holzeinlagerungen gegliedert. Zwei hier entnommene Holzproben ( $\mathrm{Hv} 6606$ und $\mathrm{Hv}$ 6607) ermöglichen eine zeitliche Einstufung dieser „Dünensandterrasse“. Ihre Aufwehung durch W-Winde begann bereits vor $3695 \pm 85$ B.P. ( $\mathrm{Hv}$ 6607), also nach der Ablagerung der Feinmaterialakkumulation im Unterlauf des Oued ez Zouara und setzte sich bis in die jüngste Zeit fort (Hv 6606; $365 \pm 65$ B.P.). Nach Ablagerung des jüngsten datierten Holzes wurde noch eine $1,3 \mathrm{~m}$ mächtige Flugsanddecke aufgeweht, auf der sich der rezente Boden entwickelte. 
Die oberen Partien der „Dünensandterrasse“ könnten bei Berücksichtigung aller Befunde etwa zu einer Zeit akkumuliert worden sein, als in Küstennähe die Feinmaterialterrasse von der älteren Flugsanddecke eingehüllt wurde (Abb. 3).

An einer Stelle, ca. $200 \mathrm{~m}$ nördlich der Nationalstraße Tabarka-Djebel Abiod (Nefza), wurde beobachtet, daß auf der hier ca. $5 \mathrm{~m}$ hohen, flächenhaft entwickelten „Dünensandterrasse " eine ca. $1 \mathrm{~m}$ mächtige braune Feinmaterialdecke ausgebreitet ist (Abb. 5). Diese dürfte nach ihrem gesamten Habitus und ihrer Höhenlage 5-6 m über Niedrigwasserbett den obersten Teil der Feinmaterialakkumulation darstellen. Die weite flächenhafte Ausdehnung der "Dünensandterrasse“ in diesem Gebiet spricht dafür, daß die Dünensande nach ihrer wohl vorwiegend äolischen Ablagerung flächenhaft fluvial überformt wurden. Im Zuge dieser Überformung wurden sie dann von dem Deck-Feinmaterial überlagert.

Die beschriebenen Aufschlüsse der Abb. 2, 5 u. 7 zeigen, daß die Ablagerung der Sedimente der Feinmaterialakkumulation und die Bildung der „Dünensandterrasse“ ungefähr im gleichen Zeitraum (zwischen ca. 5000 B.P. und 300 B.P.) erfolgt sein kann. Nicht auszuschließen ist, daß die Ablagerung der Feinmaterialakkumulation, wie schon in $\mathrm{Ka}-$ pitel 2 a. erwähnt, in zwei Phasen stattfand: in einer ersten vor Ablagerung der ältesten Teile der „Dünensandterrasse“ und in einer zweiten im Schlußabschnitt der Aufwehung der ,Dünensandterrasse“.

Mit der genaueren Untersuchung der Feinmaterialakkumulationen in den von uns besuchten Oueds ist die von FrEGIEN (1971: 145) vermutete Altersstellung der „Auelehme“, die nach ihm in den letzten 3000 Jahren gebildet worden sein müssen, modifiziert und abgesichert worden. Nach unseren Ergebnissen muß ihre Bildung zwischen etwa 5000 B.P. und der jüngeren Neuzeit erfolgt sein. Diese Befunde passen zu den Ergebnissen von Gobert \& VAufrey (1932), die in den Dünen von Ouchtata (also westlich unseres Untersuchungsgebietes bzw. in unserem Arbeitsgebiet) bedeutende prähistorische Wohnanlagen gefunden haben. Zwischen Tabarka und dem Cap Serrat fand GotTis (1953) ebenfalls zerstreut liegende, prähistorische Wohnanlagen, was auf eine frühe Besiedlung dieses Gebietes hindeutet. Vielleicht erklären sich hierdurch die recht häufigen Funde von Holzkohlestückchen in den Feinmaterialakkumulationen einerseits und von $3-5 \mathrm{~cm}$ dicken Holzkohlebändern in der „Dünensandterrasse“ andererseits. Debazac (1969: 31) fand Spuren einer Mikro-Feuerstein-Industrie nahe Babouch (nördlich Ain Draham) am Majen Khangout. Über die Siedlungstätigkeit in der historischen Epoche berichten GsELL (1921) und Mensching (1974) ausführlich.

\section{c. Altere Schotterakkumulationen und Schotterterrassen}

An vielen Stellen der untersuchten Täler liegt die Feinmaterialakkumulation bzw. das braune auelehmartige Feinsediment locker gelagerten, gut geschichteten älteren Sandsteinschottern auf, die in eine braune, sandig-lehmige Matrix eingebettet sind. Im Niedrigwasserbett konnten diese Schotter allerdings unterhalb des mittleren Mittellaufes nirgends angetroffen werden; hier dominiert im Niedrigwasserbett rezenter Schwemmsand. Die Schotter unter dem auelehmartigen Feinsediment entsprechen ohne Zweifel Fregiens (1971: 147) unterster Terrasse (womit er den untersten Schotterkörper meint, der allerdings für sich allein keine morphologische Terrasse bildet).

Die Auflagerungsfläche der flußbettnahen Sandsteinschotter liegt manchmal nur etwas über der Niedrigwasserbetthöhe auf Anstehendem (Abb. 4), manchmal bis 4-5 m über dem Niedrigwasserbett. Zusätzlich zu den dargelegten Befunden bot in einem Falle ein Brunnen, der auf der 5-6 m über Niedrigwasserbett entwickelten Oberfläche der Feinmaterialakkumulation am Fuß des Djebel Bou Lahia nahe der Lokalität Si. Salem (unte- 
rer Mittellauf des Oued Malah) angelegt war, Einblick in den Aufbau der jüngeren Akkumulationen: Hier stand der locker gelagerte grobe Sandsteinschotter ca. 2,5 m unter der Oberfläche an, wurde also von ca. 2,5 m mächtigen Feinsedimenten überlagert. An anderer Stelle (oberer Mittellauf des Oued Malah) liegt Sandsteinschotter $10 \mathrm{~m}$ über Niedrigwasserbett auf einem Mergel-Felssockel, an mehreren anderen Stellen konnten Schotter erst $25 \mathrm{~m}$ über dem Niedrigwasserbett auf einem Felssockel (Abb. 2), an drei anderen Stellen erst ab ca. $40 \mathrm{~m}$ über Niedrigwasserbett angetroffen werden (Abb. 4). Lagert der Sandsteinschotter auf $25-40 \mathrm{~m}$ hohen Felssockeln, so läßt er sich bis in $45-50 \mathrm{~m}$ über Niedrigwasserbett hinauf verfolgen.

Im Madene-Tal laufen pedimentartige Formen in ca. $40 \mathrm{~m}$ Höhe über Niedrigwasserbett (Abb. 4) bzw. in 10-12 m über Niedrigwasserbett aus. In 6-8 m über Niedrigwasserbett findet sich im Oued Malah (oberer Mittellauf) im Sandsteinschotter ein Erosions-Zwischenniveau.

Bisher ist es nicht gelungen, mit Hilfe von Aufschlüssen nachzuweisen, daß es sich bei den in verschiedenen Höhen angetroffenen Sandsteinschottern um mehrere, voneinander trennbare Akkumulationskörper handelt.

Allerdings scheinen uns doch einige Befunde dafür zu sprechen, daß es zwei, vielleicht auch drei Akkumulationsphasen von Schottern gegeben hat:

1. Für eine Mehrphasigkeit der Aufschotterung könnte vor allem der Umstand sprechen, daß mehrfach, wie erwähnt, unter Schotterkörpern Felssockel in Höhen um $40 \mathrm{~m}$, $25 \mathrm{~m}, 9-10 \mathrm{~m}, 4 \mathrm{~m}$ und etwa in Höhe des heutigen Niedrigwasserbettes gefunden wurden. Die z. T. recht ebenen, unterschiedlich hohen Felssockel wurden mehrfach direkt übereinander gefunden (z. B. Abb. 4). Hieraus ist zumindest zu schließen, daß es mehrere Eintiefungsphasen der Flüsse gegeben hat, die mit der Entstehung von im Fels ausgebildeten Talböden verbunden waren. Da das Untersuchungsgebiet in Meeresnähe liegt und hier aus verschiedenen Befunden mehrere Meeresspiegelschwankungen nachweisbar sind (Kap. 2.d.), ist die Vorstellung mehrerer zeitlich voneinander $\mathrm{zu}$ trennender Akkumulationsphasen nicht unwahrscheinlich.

2. Eine recht junge Aufschotterung ist im Bereich des Oberlaufs des Oued Malah zu beobachten. Hier hat ein Nebental vor nicht allzu langer Zeit einen ca. $8 \mathrm{~m}$ mächtigen, locker gelagerten Schotter-Schwemmfächer gegen das Haupttal vorgeschüttet, der etwa in Höhe des heutigen Niedrigwasserbettes beginnt. Diese Beobachtungen scheinen dafür zu sprechen, daß sich zumindest ein jüngerer, locker gelagerter Schotterkörper mit brauner, lehmiger Matrix, der an die Taltiefenlinien gebunden ist, von einem oder mehreren älteren Schotterkörpern auf hoch über den Tälern gelegenen Felssockeln unterscheiden läßt.

Die Schotter-Akkumulationen des untersuchten Talsystems lassen sich in diesem Arbeitsgebiet allerdings nicht mit im Anstehenden entwickelten Abrasionsterrassen in Verbindung bringen, da in Meeresnähe verkalkte quartäre Dünen dominieren, die ihrerseits von rezentem Dünensand überweht und überlagert werden.

Für eine absolute Datierung der Sandstein-Schotter ergaben sich keine Anhaltspunkte. Neben den beschriebenen Niveaus fielen noch Verflachungen bei $70-75 \mathrm{~m}$, bei $140-145 \mathrm{~m}$ (Djebel Bou Lahia) und bei $255 \mathrm{~m}$ ü.M. (Djebel bou Lahia) auf, die nicht näher untersucht wurden.

d. Kalkzementierte Dünen, alte Meeresniveaus und Abrasionsterrassen am Unterlauf des Oued ez Zouara, am Cap Negro und am Cap Serrat

Im Mündungsgebiet des Oued ez Zouara ist südöstlich der eigentlichen Küste eine ca. $0,8 \mathrm{~km}$ lange, seichte Lagune entwickelt, die vom Oued ez Zouara durchflossen wird. Bei Ebbe fallen weite Teile dieser Lagune trocken. Das Oued ez Zouara gelangt über einen schmalen Kanal in das Meer, der infolge von Strandversetzung und durch die bei Flut 
vom Meer herantransportierten Sandmassen zeitweilig vom Meer durch einen kleinen Sandwall abgetrennt wird. Eine von rezenten Flugsanden und Kupsten bedeckte Strandplatte erhebt sich nördlich und südlich der Lagune etwa $1 \mathrm{~m}$ über Niedrigwasserstand des Meeres. Nur geringfügig $(0,5-1,0 \mathrm{~m})$ höher liegt die Oberfläche der in Abb. 3 dargestellten Feinmaterialakkumulation am SE-Rand der Lagune. Sie wird vom Hochwasser nicht mehr erreicht. $\mathrm{Da}$ die Feinmaterialakkumulation mit ihrer Oberfläche vermutlich einmal auf einen etwas höheren (?) Meeresspiegel eingestellt gewesen war, ist sie mit ihrem NEEnde als fossiles, niedriges Kliff zu deuten, das später im Zuge einer äolischen Tätigkeit durch Flugsande fossilisiert wurde.

Am Cap Serrat bei Sedjenane wurden bei 2,0-2,5 m ü. M. in durch Kalk verkitteten Dünensanden kleinräumige Abrasionsflächen gefunden, die ebenfalls als fossil anzusehen sind. Bis $0,5 \mathrm{~m}$ ü. M. reichen in diesen kalkzementierten Dünen mit Terra rossa-Material ausgefüllte VerwitterungsSchlotten herab. Außerdem gibt es hier im nördlichen Teil einen $2 \mathrm{~m}$ ü. M. gelegenen AbrasionsFelssockel mit $1 \mathrm{~m}$ mächtigen Brandungsgeröllen unter fossilen kalkzementierten Küstendünen, die an der Oberfläche von mit Terra rossa-Material gefüllten Orgeln besetzt sind. Hier ist nicht die ganze Düne durch Kalk verkittet, sondern nur die 4-5 m dicke Außenhaut, wie durch frische Aufschlüsse ersichtlich ist.

Im küstennahen Teil des Oued ez Zouara sind durch Kalk verkittete fossile Dünen wie am Cap Serrat - die beherrschenden Formen. Wie am Cap Serrat haben sich nach oder während der Zementierung der Dünen durch Kalk auch hier 1,5-2 m tiefe Schlotten ausgebildet, die mit einem tief rot gefärbten Terra rossa-Material gefüllt sind. Rötliche Bodensedimente, die dem Terra rossa-Material in den Schlotten ähneln, wurden auf den kalkverkitteten Dünen bis in Höhen von 70-90 m ü.M. gefunden (Abb. 9). Hier konnten auch stellenweise Osteokolle in dem roten Verwitterungsmaterial angetroffen werden.

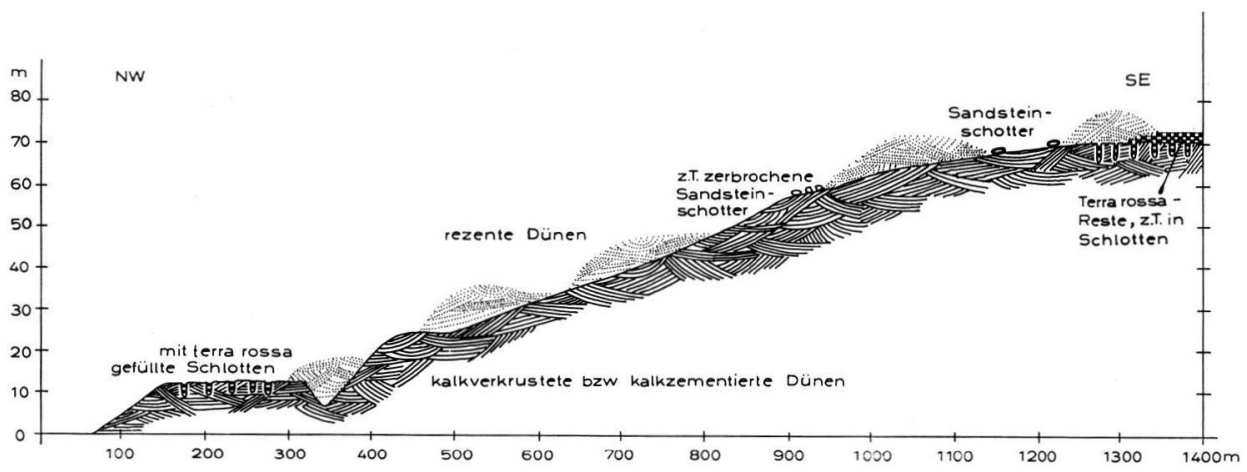

Abb. 9: Von rezenten Dünen überwehte, kalkzementierte ältere Dünen mit Terra rossa-Resten in $1-2 \mathrm{~m}$ tiefen Schlotten. In Küstennähe ist ein $12,5-13,5 \mathrm{~m}$ hohes Erosionsniveau (Abrasionsniveau?) entwickelt.

Da die kalkverkitteten Dünen stellenweise bis unter den Meeresspiegel verfolgbar sind (Cap Serrat), ist der Schluß zulässig, daß die Dünen zu einer Zeit aufgeweht wurden, als der Meeresspiegel tiefer lag als heute (vgl. die Befunde von Moseley 1965: 181 in Libyen ${ }^{4}$ ). Das kann in erster Linie während des Maximums einer Kaltzeit oder auch während der Übergangsphasen von einer Kaltzeit zu einer Warmzeit (oder umgekehrt) geschehen sein (vgl. auch Rohdenburg \& SABelberg 1973). In einem solchen Zeitabschnitt

4) In einer solchen Zeit können auch steil abtauchende, 4-5 m dicke Sandstein-Hangschuttdecken entstanden sein, die ca. 1,5 km südöstlich der Küste auf der E-Seite des Oued ez Zouara gefunden wurden. Diese reichen hier auch bis mindestens ans Niedrigwasserbett hinab. 
lagen weite Strandflächen trocken, die als bevorzugtes Ausblasungsgebiet für Dünensande in Frage kommen.

Die kalkzementierten Dünen im Mündungsgebiet nördlich und südlich des Oued ez Zouara weisen einige Verflachungen und Hangknicke auf (Abb.9); diese dürften aber keine überregionale Bedeutung haben. Das 12,5-13,5 m hohe Niveau in Küstennähe ist als deutliches Erosionsniveau (Abrasionsterrasse?) entwickelt, wie sich aus der Kappung der kalkzementierten Dünensandlagen entnehmen läßt. Dieses Niveau könnte bei einem gegenüber heute höheren Meeresspiegel etwa zur selben Zeit entstanden sein, als weiter landeinwärts Sandsteinschotter auf hohen Felssockeln aufgeschüttet wurden. In einigen Fällen wurden auch Felsterrassen in ca. 8-10 $\mathrm{m}$ Höhe über dem Niedrigwasserbett gefunden.

Die gleiche Beziehung zu einem höheren Meeresspiegelstand kann auch bei den höheren, in Küstennähe entwickelten Niveaus und den im Inland dominierenden Niveaus (Felssockelhöhen und Schotterakkumulationshöhen) bestanden haben. Allerdings scheinen uns die sporadisch entwickelten Verflachungen auf den kalkverkitteten Dünen nicht ausreichend aussagekräftig für die Ableitung höherer, über längere Zeiträume konstanter Meeresspiegelstände in ganz bestimmten absoluten Höhen zu sein.

Die höchsten kalkverkitteten Dünen im Zouara-Gebiet wurden bei $125-130 \mathrm{~m}$ ü.M. angetroffen. Sandsteinschotter über den fossilen Dünen, auf die heute junge lockere Flugsande und Dünen aufgeweht werden, konnten bei $125 \mathrm{~m}$, bei $110 \mathrm{~m}$, bei $95-100 \mathrm{~m}$ und bei $60-70 \mathrm{~m}$ ü.M. gefunden werden. Es ist nicht auszuschließen, daß die Sandsteinschotter vom direkt benachbarten Bergland über lokale Gerinne herantransportiert worden sind.

Vergleicht man die Niveaus am Oued ez Zouara mit den Abrasionsniveaus über kalkzementierten Dünen am Cap Serrat, so zeigt sich, daß in beiden Gebieten ein 11,5-12,0 m hohes, bzw. 12,5-13,5 m hohes Niveau auftritt. Weitere Übereinstimmungen wurden zwar zwischen diesen beiden Gebieten nicht festgestellt, jedoch ergaben sich Ubereinstimmungen zwischen dem Formenschatz am Cap Serrat und am Cap Negro. In beiden Fällen

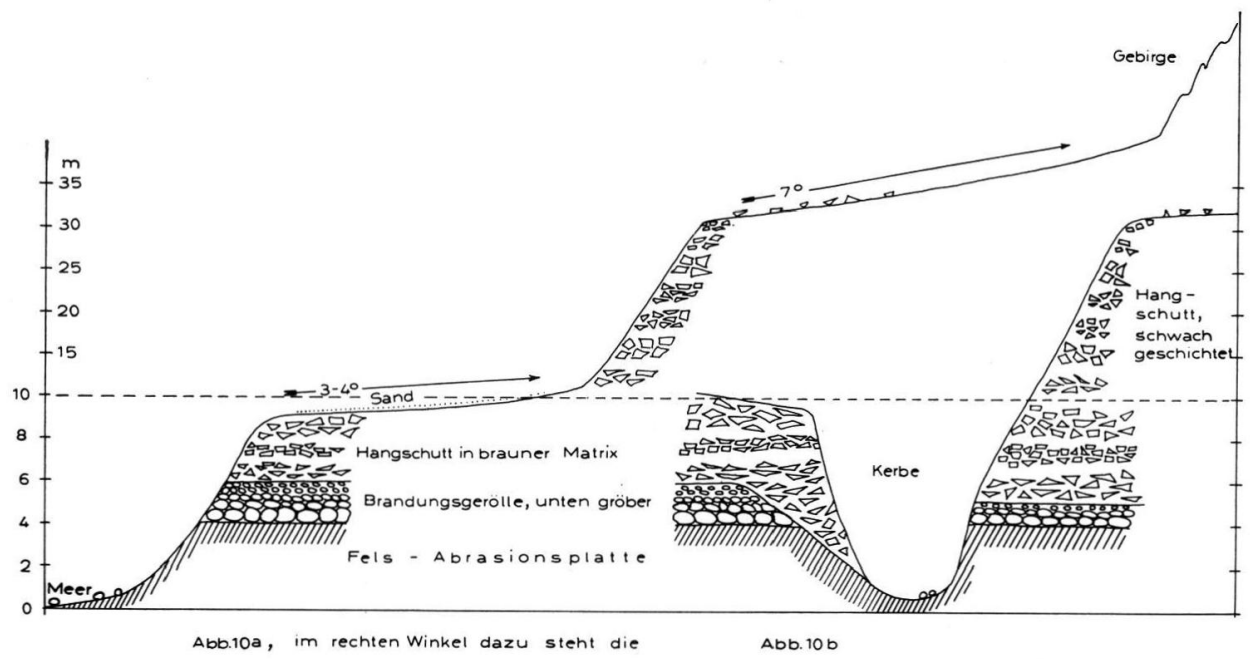

Abb. 10 a u. b: Abrasionsniveau mit ca. $2 \mathrm{~m}$ mächtigen Brandungsgeröllen am Cap Negro (nördl. Nefza). Die Brandungsgerölle werden von schwach geschichteten Schuttmassen überlagert, die sich glacisartig vor dem Gebirge ausdehnen. In 9-10 m ü.M. Erosionsniveau im Schuttglacis, rezente Kerbe auf Meeresniveau eingestellt. 
finden sich Abrasions-Felssockel in 2 bzw. $4 \mathrm{~m}$ ü.M. mit Brandungsgeröll-Auflagen (CASTANY 1953). Diese werden am Cap Serrat von fossilen kalkzementierten Dünensanden mit eingelassenen Terra rossa-Schlotten und am Cap Negro von fossilen schwach geschichteten, > $20 \mathrm{~m}$ mächtigen Schwemmschuttmassen überlagert (Abb. $10 \mathrm{a} \mathrm{u}$. b). Wir vermuten, daß die bei 2 bzw. $4 \mathrm{~m}$ ü.M. gelegenen Abrasionsplatten mit ihren Brandungsgeröllen möglicherweise einem interstadialen oder dem letzten interglazialen Meereshochstand zugeordnet werden können. Die jüngeren auflagernden Schwemmschutt-Sedimente könnten danach am Cap Negro in einer Zeit kräftiger mechanischer Verwitterung, vermutlich in der Würm-Kaltzeit, entstanden sein.

\section{Ergebnisse}

Im nordtunesischen Untersuchungsgebiet lassen sich unterscheiden:

- eine Feinmaterialakkumulation bis zu einer Höhe von 5-6 m über Niedrigwasserbett mit einem Erosionsniveau bei 2-2,5 m;

- eine 6-12 m mächtige „Dünensandterrasse“;

- ein Schotterkörper, der bis zu einer Höhe von 6-10 m über dem Niedrigwasserbett hinaufreicht;

- kalkzementierte Dünen mit Terra rossa-Schlotten bei 12,5-13,5 m ü.M. in Meeresnähe;

- eine oder mehrere Schotterterrassen mit Felssockeln zwischen 25-40 m über Niedrigwasserbett;

- kalkzementierte Dünen mit roten Bodensedimenten und einzelnen Sandsteinschottern bei $70-90 \mathrm{~m}$, stellenweise sogar bei $130 \mathrm{~m}$ relativer Höhe über Niedrigwasserbett.

Ein Vergleich mit den Untersuchungsergebnissen von Fregien (1971) zeigt, daß der von ihm als "Auelehm“ gedeutete Feinsedimentkörper unserer Feinmaterialakkumulation bis in Höhe des Erosionsniveaus bei 2-2,5 m über Niedrigwasserbett entsprechen dürfte. Die Schotterkörper seiner 1. und 2. Schotterterrasse sind wahrscheinlich mit unserem jüngsten Schotterkörper zu parallelisieren. Das von ihm beschriebene Niveau der 2. Schotterterrasse stellt nach unseren Untersuchungen das obere Niveau unserer Feinmaterialakkumulation dar. Eine unserer älteren Schotterterrassen könnte mit der 3. Schotterterrasse von FREGIEN korrelieren.

Wie zwei ${ }^{14} \mathrm{C}$-Datierungen ergaben, wurden die Feinmaterialsedimente im jüngeren Holozän und vermutlich auch noch in historischer Zeit abgelagert: in einem Zeitraum um etwa 5000 B.P. bis nach 700 B.P. Es ließ sich bisher nicht eindeutig klären, ob dieses Sediment während eines kontinuierlichen Akkumulationsvorganges, z. B. während einer anhaltenden Phase rückschreitender Akkumulation vom Meer zum Landesinneren, entstand, oder ob dieses Sediment in mehreren Phasen abgelagert wurde. Die Untersuchungen von Bos (1971), der für den älteren Abschnitt des Holozäns eine Phase intensiver Bodenbildung wahrscheinlich macht, und der Aufbau der Feinmaterialakkumulation aus graubraunen bis braunen, feinkörnigen Sedimenten lassen ihre Herkunft aus Abtragungsprodukten älterer Böden wahrscheinlich werden.

Ein Vergleich mit Untersuchungsergebnissen aus Zentraltunesien (MOLLE \& BROSCHE

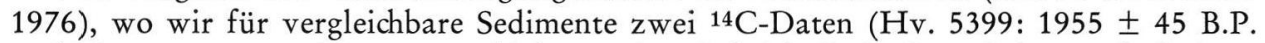
und Hv. 5394: $1100 \pm 65$ B.P.) erhielten, zeigt, daß sich die in den mittleren Abschnitten der Flußläufe in Nordtunesien abgelagerten Sedimente und die Feinmaterialakkumulation in Zentraltunesien zeitlich überschneiden können. Die Feinmaterialsedimente direkt an der Küste im nordtunesischen Untersuchungsgebiet scheinen dagegen ein höheres Alter als die Feinmaterialakkumulation in Zentraltunesien zu besitzen. 
Jüngere als frühholozäne Bodenbildungsphasen (Bos 1971) sind in unserem nordtunesischen Arbeitsgebiet durch mehrere Bodenhorizonte in der „Dünensandterrasse“ belegt, die im küstennahen Bereich des Untersuchungsgebietes nach zwei ${ }^{14} \mathrm{C}$-Daten seit etwa 4000 B.P. entstanden ist. Die Sedimente dieser Terrasse deuten außerdem auf eine seit dem jüngeren Holozän bis heute zeitweilig auftretende, starke äolische Aktivität in Küstennähe hin. Die teilweise zeitliche Überschneidung der Ablagerung dieser äolischen Sedimente mit derjenigen der Feinmaterialakkumulation läßt eine Gleichzeitigkeit fluviatiler und äolischer Ablagerungsprozesse, möglicherweise aber auch ein Nacheinander dieser Vorgänge in mehrfachem Wechsel im Untersuchungsgebiet vermuten, da die Feinmaterialakkumulation auch in mehreren, zeitlich voneinander $\mathrm{zu}$ trennenden Phasen entstanden sein kann. Das häufige Vorkommen von Holzkohlebändern in den Sedimenten der „Dünensandterrasse" und Feinmaterialterrasse deutet auf eine starke Siedlungstätigkeit im jüngeren Holozän hin, die auch durch archäologische Untersuchungen belegt ist (GOBERT \& VAUFREY 1932; GotTIS 1953).

Eine Datierung des jüngsten Schotterkörpers ist in Nordtunesien im Unterschied zu Zentraltunesien, wo mehrere ${ }^{14} \mathrm{C}-$ Daten vorliegen (Molle \& BROsche 1976), bisher nicht möglich. Eine Korrelation des jüngsten Schotterkörpers in Nordtunesien mit der Hauptakkumulation in Zentraltunesien (MOLLE \& BROSCHE 1976) scheint uns möglich zu sein, $\mathrm{da}$ es sich in beiden Untersuchungsgebieten um die jüngste, überall verbreitete Schotterakkumulation handelt. Zur Zeit der Bildung des Schotterkörpers ist eine Phase starker mechanischer Gesteinsaufbereitung im Untersuchungsgebiet anzunehmen, worauf auch mächtige Hangschutt- und Schwemmschuttmassen an verschiedenen Stellen hinweisen. Die Lage des Schotterkörpers zu den datierten jüngeren Sedimenten läßt ein würmzeitliches Alter der Schotter in Nordtunesien vermuten. In Zentraltunesien ist diese zeitliche Einstufung durch ${ }^{14} \mathrm{C}$-Alter zwischen ca. 10000-30000 B.P. belegt. Wie die Untersuchungen von Dresch, Rondeau \& El Aouani (1960), Guillien \& Rondeau (1966), Bos (1971) und unsere Untersuchungen in Zentraltunesien (Molle \& Brosche 1976) zeigen, ist in den Gebirgen Tunesiens in der letzten Kaltzeit mit Prozessen der Solifluktion, Nivation und Aufbereitung des Gesteins durch Frostwirkung zu rechnen.

Bisher ließ sich nicht feststellen, in welcher Beziehung die kalkzementierten Dünen in unmittelbarer Küstennähe bei 12,5-13,5 m über Niedrigwasserbett zu dem genannten jüngsten Schotterkörper stehen. Ungeklärt bleibt auch, ob die Reste einer roten Bodenbildung auf diesen Dünen möglicherweise einer Phase rötlicher Bodenbildung in Zentralbzw. Südtunesien (Giessner 1964: 146; Mensching 1974; Brosche \& Molle 1975; Molle \& Brosche 1976) entsprechen könnte. Hinweise auf Korrelationsmöglichkeiten der Schotterlagen auf Felssockeln bei $25-40 \mathrm{~m}$ und der Verebnungsflächen mit einzelnen Sandsteinschottern auf kalkzementierten Dünen bis in Höhen um $130 \mathrm{~m}$ über Niedrigwasserbett in Nordtunesien einerseits mit Schotterniveaus oberhalb der Hauptakkumulation in Zentraltunesien andererseits ließen sich bisher nicht finden.

A n merkung: Die vorgelegte Arbeit ging aus Geländestudien hervor, die auf einer Reise nach Tunesien im Sommer 1974 durchgeführt wurden. Für die Finanzierung dieser Reise sind wir dem Fachbereich 24, Geowissenschaften, der FU Berlin zu Dank verpflichtet. Herrn Prof. Dr. M. A. Geyh, Hannover, und seinen Mitarbeitern danken wir für die Datierung einer Reihe von ${ }^{14} \mathrm{C}$ Proben.

\section{Schriftenverzeichnis}

Bos, R. H. G. (1971): Quaternary Evolution of a mountainous area in N. W. Tunisia. A geomorphological and pedological analysis. - Publicaties van het Fysisch-Geografisch en Bodemkundig Laboratorium van de Universiteit van Amsterdam, Nr. 19: 176 S.; Amsterdam. 
Brosche, K.-U. \& Molle, H.-G. (1975): Morphologische Untersuchungen im nördlichen MatmataVorland (nördliche Djeffara, Südtunesien). - Eiszeitalter u. Gegenwart, 26: 218-240; Ohringen.

- (1976): Geomorphologische und klimageschichtliche Studien in Süd- und Zentraltunesien. Z. Geomorph., N. F., Suppl. Bd. 24: 149-159; Berlin - Stuttgart.

Castany, G. (1953): Le Tyrrhénien de la Région de Bizerte. - Bull. Soc. des Sci. natur. de Tunisie, 4, 1-4: 169-175; Tunis.

Debazac, E.-F. (1959): La végétation forestière de la Kroumirie. - Ann. de l'école nat. des eaux et forêts et de la station de Rech. et expériences, 16 (2): 1-113; Nancy.

Dresch, J., Rondeau, A. \& El Aouani, M. (1960): Observations sur les dépots de versants et les terrasses climatiques en Tunisie. - C. R. Soc. Géol. France, 6: 137-139; Paris.

Fregien, W. (1971): Das Kroumir- und Mogodbergland im Nordtunesischen Küstentell. Grundlagen und Wirksamkeit der Morphodynamik in einer mediterran-humiden Landschaftsregion des Maghreb. - Jb. d. geogr. Ges. zu Hannover, Sonderh., 5; Hannover.

GIEssner, K. (1964): Naturgeographische Landschaftsanalyse der tunesischen Dorsale (Gebirgsrücken). - Jb. d. geogr. Ges. Hannover f. 1964; Hannover.

Gobert, E.-G. \& VAufrey, R. (1932): Deux gisements extrêmes d'Ibéromaurusien. - L'Anthrop., 42; Paris.

Gоттіs, Ch. (1953): Sur l'âge des dunes de la région d'Ouchtata (Nefza, Tunisie Septentrionale). - Bull. Soc. natur. de Tunisie, 6 (1-4): 223-226; Tunis.

Gsell, S. (1921): Histoire ancienne de l'Afrique du Nord. I: Les conditions du développement historique; Paris.

Guillien, Y. \& Rondeau, A. (1966): Le modelé cryonival de la Tunisie centrale et septentrionale. - Ann. de Géogr., 409: 257-267; Paris.

JaUzein, A. (1959): Les terrasses alluviales en Tunisie septentrionale. - C. R. som. et Bull. Soc. Géol. France, 2: 31-32; Paris.

Mensching, H. (1958): Glacis - Fußfläche - Pediment. - Z. Geomorph., N. F., 2: 165-186; Berlin - Stuttgart.

- (1963): Morphologie des südtunesischen Schichtstufenlandes. - Bull. Soc. hellén. Géogr., 152-161; Athen.

- (1974): Tunesien, eine geographische Landeskunde. - Wiss. Länderkunden, 1, 2. Aufl.: 281 S., 18 Fig., 9 Ktn., 14 Bildtaf.; Wiss. Buchgesellschaft Darmstadt; Darmstadt.

Molle, H.-G. \& Brosche, K.-U. (1976): Morphologische und klimageschichtliche Untersuchungen im südöstlichen Vorland des Djebel Chambi und des Djebel Mrhila in Zentraltunesien. - Die Erde, 107, H. 2/3: 81-145; Berlin.

Moseley, F. (1965): Plateau calcrete, calcreted gravels, cemented dunes and related deposits of the Maalegh-Bomba region of Libya. - Z. Geomorph., N. F., 9: 166-189; Berlin - Stuttgart.

Roudenburg, H. \& SAbelberg, U. (1973): Quartäre Klimazyklen im westlichen Mediterrangebiet und ihre Auswirkungen auf die Relief- und Bodenentwicklung. - Catena, 1: 71-180; Gießen. 